\title{
Screening for congenital hypothyroidism: the first decade
}

\author{
N D BARNES
}

\author{
Addenbrooke's Hospital, Cambridge
}

The horror of cretinism and the joy of its treatment can never have been expressed more vividly than by William Osler, writing in 1897 soon after the effect of thyroid replacement was recognised. 'No type of human transformation is more distressing to look at than an aggravated case of cretinism. The stunted stature, the semi-bestial aspect, the blubber lips, retroussé nose sunken at the root, the wide-open mouth, the lolling tongue, the small eyes half-closen with swollen lids, the stolid expressionless face, the squat figure, the muddy, dry skin, combine to make the picture of what has been well termed the "pariah of nature". . . Not the magic wand of Prospero or the brave kiss of the daughter of Hippocrates ever effected such a change as that which we are now enabled to make in these unfortunate victims, doomed heretofore to live in hopeless imbecility, an unspeakable affliction to their parents and their relatives.' 1

Not until 60 years later was it first reported that although thyroid replacement treatment did indeed abolish the physical manifestations of congenital hypothyroidism, unless it was started within the first few weeks of life serious intellectual and neurological deficits frequently persisted. ${ }^{2}$ This remained a matter of controversy but was supported by increasing weight of evidence. In 1972 a study from Pittsburgh, for instance, showed that treatment started before the age of 3 months resulted in a mean intelligence quotient (IQ) of 89 , between 3 and 6 months of 70 , and after 6 months of only $54 .^{3}$

Prompt diagnosis was therefore essential but proved impossible to achieve on clinical grounds alone. Few infants showed diagnostic features of hypothyroidism at birth - the early manifestations were subtle and non-specific, and the stigmata of 'aggravated cretinism' developed only slowly. For these reasons, even in the best circumstances, less than half were identified before 3 months of age. ${ }^{4}$

The potential benefit of neonatal screening was evident, and when radioimmunoassays became available for measurement of thyroxine and later thyroid stimulating hormone in small samples of serum or whole blood spotted on to filter paper, pilot programmes were organised without delay. In 1973 screening was started in Pittsburgh using umbilical cord serum thyroid stimulating hormone, in Quebec using filter paper thyroxine, and in Belgium using serum thyroid stimulating hormone measurement. These and many other programmes soon proved that congenital hypothyroidism could indeed be detected biochemically in the newborn period (almost invariably before it was suspected clinically) with great reliability and acceptably low cost. ${ }^{5} 6$

In the United Kingdom the financially constrained National Health Service was slow to support this advance in preventive medicine. By 1979, when the first NHS funded regional programme started (in East Anglia), there was already national screening in six European countries and in much of North America. In 1981, however, a strong recommendation (but no extra money) was conveyed to health authorities, and in 1983 screening reached all parts of the country. In addition, the Medical Research Council funded the establishment of a National Register of Congenital Hypothyroidism based at the Institute of Child Health in London.

What has been learned from a decade of screening and what remains to be learned?

\section{Perinatal thyroid function}

The observations made in screening programmes, in combination with clinical and experimental studies, have clarified many aspects of normal and abnormal perinatal thyroid development and function. From the work of Fisher and others has evolved the useful concept that thyroid axis maturation occurs in three partially overlapping phases, during each of which developmental errors may occur. ${ }^{7}$ Permanent abnormalities generally result from genetic or embryo- 
logical defects while transient disorders are more commonly due to delays in functional maturation (Table).

Neither thyroid hormones nor thyroid stimulating hormone cross the placenta in appreciable concentrations; the fetal axis therefore develops independently of maternal influence. In the first trimester of pregnancy the fetal thyroid and pituitary glands differentiate anatomically and functionally. The embryological migration of the thyroid anlage from its origin to its cervical site is an accident prone journey, errors in which cause the related disorders of ectopia, hypoplasia, and aplasia. These together are the most common and important causes of permanent congenital hypothyroidism and are responsible for a spectrum of dysfunction from the mildest to the most severe. The several genetic defects in thyroid hormone biosynthesis are autosomal recessive disorders usually associated with relatively mild goitrous hypothyroidism. Pituitary dysplasia is rare, it may occur as an isolated defect or in association with other midline malformations and often presents with other manifestations of hypopituitarism such as hypoglycaemia or, in the male, micropenis. Hypothalamic maturation is a later event in fetal life; developmental disorders are rare and often associated with other problems such as blindness in septo-optic dysplasia.

Around mid-gestation the integrity of the hypothalamic-pituitary portal system is established and functional maturation of the thyroid axis can occur but in man this process is not completed until after birth. There is a gradual rise in fetal thyroid hormones and thyroid stimulating hormone but concentrations of the active monodeiodinated thyroxine derivative, triiodothyronine, remain low while those of the inactive metabolite, reverse triiodothyronine, are relatively high. At birth dramatic changes occur. There is a sharp rise in thyroid stimulating hormone to reach a peak 30 minutes after birth. This elicits an appropriate release of thyroid hormones. Also within six hours there is a switch from inner ring to outer ring monodeiodination of thyroxine, which results in a four to sixfold rise in triiodothyronine.

These remarkable mechanisms, which spare thyroid hormone effect in the fetus and then amplify it immediately after birth, suggest one reason why early postnatal hormone replacement is so effective. The other major factor is probably the late brain growth spurt in the human compared with other species, which permits the critical period for treatment to extend into the early postnatal weeks. ${ }^{8}$ Congenital hypothyroidism is thus a condition in which, to a large extent, the 'clock starts at birth'.

In the preterm infant similar changes occur but they are of a lesser degree. All preterm infants therefore have some degree of hypothyroxinaemia and they are also more prone to functional aberrations. $^{79}$

Table Developmental disorders of thyroid function

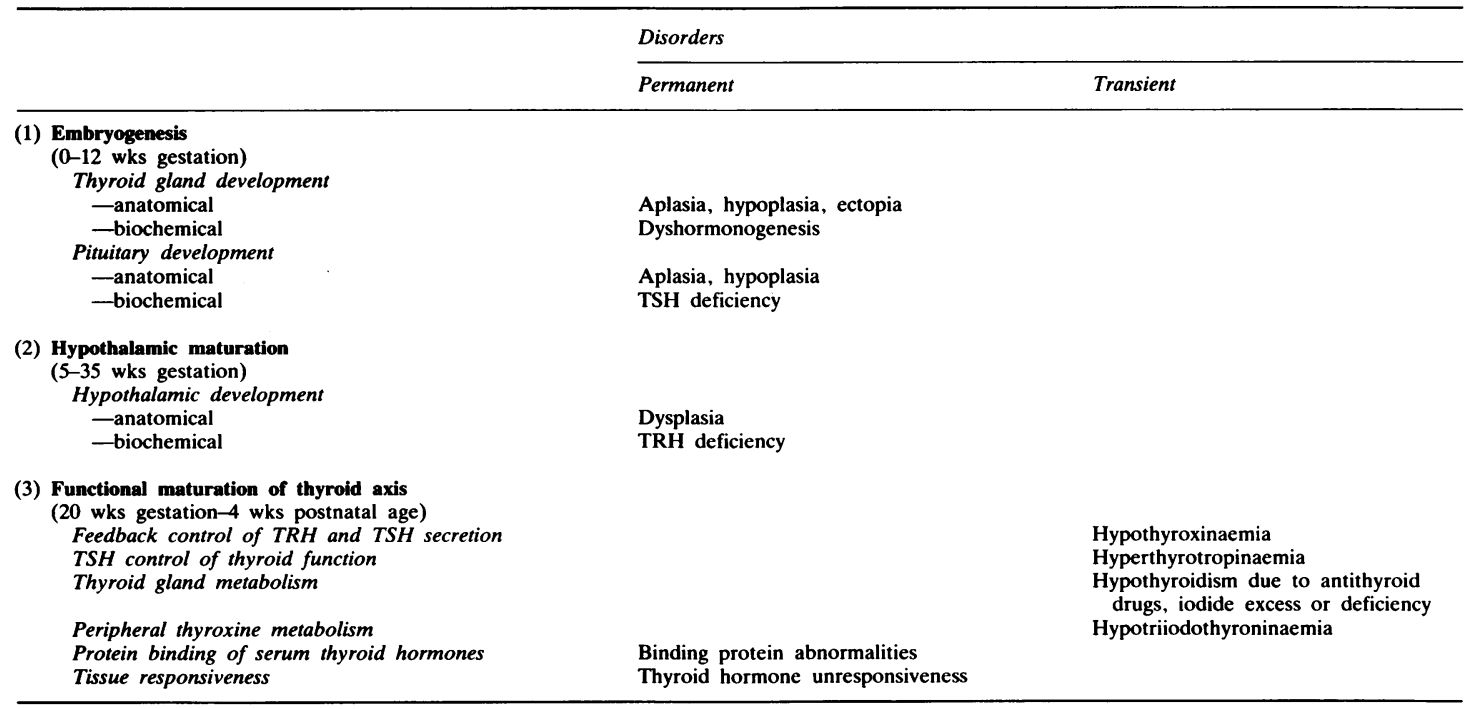

Adapted from Fisher and Klein. ${ }^{7}$

TSH=thyroid stimulating hormone; TRH=thyrotrophin releasing hormone. 


\section{Screening methods}

Screening tests must be acceptable and must combine maximum discrimination with minimum cost and inconvenience. Cord blood screening for congenital hypothyroidism offered the earliest postnatal diagnosis, but to collect an additional blood sample for this purpose alone was inevitably complex and expensive. ${ }^{10}$ By good fortune, the optimum period for screening after the immediate postnatal perturbation of the axis proved to coincide with that for screening for disorders of amino acid metabolism, the third to seventh days of life, so the two could be combined and established systems of sample collection used.

Measurement of thyroxine alone proved inadequate as many infants with 'compensated' primary hypothyroidism had values within the normal range so that approximately $30 \%$ of cases were missed. ${ }^{6}$ To measure both thyroxine and thyroid stimulating hormone on all specimens was ideal but expensive. The alternatives therefore were a primary thyroid stimulating hormone measurement or a primary thyroxine measurement with supplementary thyroid stimulating hormone determination on the lower thyroxine values, the cut off varying in different programmes from 3 to $20 \%$. The former method was more widely adopted in Europe ${ }^{6}$ and the latter in the USA. ${ }^{5}$ The pros and cons of these two approaches have been and are still widely debated. ${ }^{911}$ Thyroid stimulating hormone $T$ measurement provides the most sensitive index of primary hypothyroidism, the condition that screening programmes are designed to detect, but secondary hypothyroidism and cases with a delayed rise in this hormone are missed. With thyroxine/thyroid stimulating hormone testing if a generous cut off point is used, only the mildest cases of compensated primary hypothyroidism are missed and infants with secondary hypothyroidism are detected. In general, recall rates are higher but costs lower with primary thyroxine than with primary thyroid stimulating hormone measurement. At present there seems little to choose between these two approaches. It may prove that primary thyroxine is more discriminating when an early test is used and primary thyroid stimulating hormone when blood sampling is delayed a few days. Improved methods for both estimations using enzyme immunoassays and more automation are under development and these in time should provide more rapid and sensitive sample analysis. ${ }^{9}$

\section{Results}

Screening showed that congenital hypothyroidism is much more common than was suspected. Retrospec- tive studies had suggested an incidence of permanent primary hypothyroidism around 1 in $7000^{4}$ but screening gave a true rate of 1 in 3500 to 4500 in many parts of America, Europe, and Asia. ${ }^{5} 69$ This wide discrepancy is at least in part due to neonatal identification of children in whom the diagnosis would previously have been acquired hypothyroidism of early postnatal onset. Many of these patients have dysplastic glands with sufficient function to tide them through the early months of life without symptoms. Although the neurological and intellectual prognosis is probably good even if treatment is delayed, the possibility of subtle damage cannot be excluded and with screening they are spared the often prolonged hiatus between the eventual onset of symptoms and the start of treatment.

Thyroid dysgenesis accounts for 80 to $90 \%$ of the cases identified; approximately one third have no detectable thyroid tissue and two thirds an ectopic or dysplastic gland. ${ }^{59}$ Dyshormonogenesis, secondary hypothyroidism, and transient cases together total $10 \%$ to $20 \%$. Secondary hypothyroidism is rare with an incidence of only 1 in 60000 to 100000 ; some severely affected infants may die from hypoglycaemia before they are screened but for those who survive the prognosis with appropriate replacement treatment is good.

Although permanent abnormalities of thyroid function have been detected with remarkable consistency world wide, transient abnormalities have shown wide local variation, with a higher incidence in Europe, (around 1 in 8000) than America (1 in $60000) .569$ It is not yet clear whether these discrepancies chiefly reflect differences in screening techniques and timing or whether they may indicate true differences due perhaps to iodine intake or other factors.

Primary thyroxine screening programmes have also shown that thyroxine binding globulin deficiency is relatively common, affecting approximately 1 in 9000 infants, with an incidence nine times greater in boys than girls due to $\mathrm{X}$ linked inheritance. ${ }^{5}$ This condition is not associated with abnormal thyroid function and it is important to be able to confirm it by direct thyroxine binding globulin measurement on the screening blood sample.

At present it is estimated that seven to nine million infants are screened annually and programmes are active or planned in most parts of the developed world.

\section{Diagnosis}

The results of positive screening tests must be confirmed on serum samples but when the screening tests are unequivocal, treatment can be started 
without further delay than necessary to collect suitable blood specimens. Estimation of the bone age gives some indication of the degree of fetal hypothyroidism that was present.

A thyroid scan with ${ }^{125}$ iodine or ${ }^{99} \mathrm{~m}_{\text {technetium }}$ shows the site and size of any thyroid tissue present; if not immediately available a satisfactory scan can be obtained after treatment is started since the thyroid stimulating hormone and therefore the thyroid uptake is only slowly suppressed. ${ }^{13}$ Although not an essential investigation, radiation exposure is low and knowledge of the anatomy may be helpful both in planning medical management and in showing the abnormality to the parents in easily comprehensible form.

When the thyroid function tests are equivocal full investigation is indicated and a clinical decision must be made on whether or not to treat. ${ }^{12}$ The interpretation of thyroid function tests in sick preterm infants poses particular problems. ${ }^{9}$ The essential is to avoid leaving untreated any infant with appreciably subnormal thyroid function in the early weeks and months of life. It is better to err in favour of treating, with early reassessment if doubt persists.

\section{Treatment}

Synthetic L-thyroxine is the preparation of choice for replacement. A suitable initial dose is 10 $\mu \mathrm{g} / \mathrm{kg} /$ per day. ${ }^{13}$ Giving this 'prohormone' rather than the more active triiodothyronine is preferable as it permits the peripheral monodeiodinating mechanisms, assuming these are intact and correctly 'set', to make fine adjustment to the amount of active hormone reaching its receptors. ${ }^{14}$ This mechanism seems to permit some leeway in dosage and accords with the observation that the relatively large replacement doses given in the past seldom caused symptomatic hyperthyroidism. In postnatal hypothyroidism adequate dosage can be judged from the patient's own thyroid axis and the correct dose defined as the smallest amount which suppresses the thyroid stimulating hormone into the normal range. In congenital hypothyroidism, however, this set point may be abnormal, presumably as a result of prenatal hypothyroidism, so excessive dosage may result from attempts to suppress completely the thyroid stimulating hormone, especially in the early months of life. ${ }^{15}$ The total serum thyroxine value should be maintained in the upper part of the normal range ${ }^{13}$ the triiodothyronine concentration is then generally also within the upper normal range for age. The use of free thyroid hormone concentrations has not yet been fully evaluated but may permit more exact dosage adjustment.

\section{Follow up and reassessment}

Regular monitoring of progress, with special attention to growth, psychomotor development, symptoms or signs of thyroid dysfunction, and biochemical thyroid function tests is essential. The latter indicate not only adequacy of dose but also compliance. A suitable regimen comprises a check one month and three months after starting replacement treatment, then three monthly through the first year, six monthly until school age, and annually until growth and development is complete. Thyroid function should also be rechecked approximately one month after any change of thyroxine dose.

It is essential to reassess the diagnosis before committing a child to lifelong replacement treatment, firstly because hypothyroidism may prove transient and secondly because all diagnostic tests are fallible. Such reassessment should be undertaken after the age of 2 years when a brief interruption of treatment will not be harmful. Reversion to chemical hypothyroid status is more rapid if triiodothyronine is substituted for thyroxine beforehand as the half life is shorter. A dose of triiodothyronine equivalent to the current thyroxine dose $(20 \mu \mathrm{g}$ triiodothyronine is approximately equipotent with $100 \mu \mathrm{g}$ thyroxine) is given for one month and then treatment is stopped for 10 to 14 days to permit thyroid function testing. ${ }^{16}$ An isotope scan can also be obtained at this time.

\section{Prognosis}

The evidence available to date amply confirms that early treatment is associated with a good prognosis. Most follow up studies have found no difference between affected children treated within the first four weeks of life and normal controls. In the large New England collaborative study the results were identical for adequately treated patients and controls at 2 years on the Bayley mental development index and at 3, 4, and 5 years in Stanford-Binet IQ assessment. Indequately treated patients showed significantly reduced IQ scores. ${ }^{13}$

Some notes of caution, however, have been sounded. The Quebec group found that although no deficit was apparent at 1 year some children showed deficiencies in hearing-speech performance scales and practical reasoning at 18 and 36 months. ${ }^{17}$ Others have found that although intelligence is normal there may be subtle deficits in motor and perceptual abilities, speech, and possibly also in behaviour and personality. ${ }^{9}$

Careful and detailed follow up into school age and well beyond will be necessary before definitive conclusions can be drawn. A remarkable improve- 
ment in IQ, with a mean increment of 21 points, was reported in affected children retested 16 to 26 years after their first test. ${ }^{18}$

\section{Cost-benefit}

For few forms of medical endeavour is a realistic assessment of cost-benefit possible and if strict financial benefit were a criterion for acceptance many established treatments would be threatened. For neonatal thyroid screening it is possible to estimate costs with some accuracy and, however the benefits are computed, the ratio is overwhelmingly favourable. ${ }^{19}$ These exercises have political uses but in human terms the contribution of screening to the prevention of handicap is without price.

\section{Problems}

All screening programmes pose problems. Some failures are inevitable. They must, of course, be kept to the absolute minimum, not least because diagnostic awareness inevitably decreases when a condition is no longer seen by practising doctors. The occasional child to slip the net may therefore suffer more than before screening was established.

The logistic problems of specimen collection, labelling, transport to the screening laboratory, handling in the laboratory, and communication of results usually generate more problems and failures than the diagnostic chemistry. This has been the experience with thyroid screening programmes. ${ }^{20}$

Remarkably few serious technical problems have arisen with the thyroxine and thyroid stimulating hormone assays which were well tried and tested before their adaptation for screening. False positive thyroid stimulating hormone assays have been reported due to the presence of cross reacting antibodies induced either by immunisation of the mother with a preparation containing rabbit serum ${ }^{21}$ or occurring spontaneously. ${ }^{22}$

Recall is another major problem. The number of unsatisfactory specimens received in the laboratory can be reduced as the collecting force, in the UK generally the midwives, gains experience but nevertheless is seldom less than 1 to $2 \%$. Recall for equivocal results on the screening tests is lower with primary thyroid stimulating hormone than with primary thyroxine screening programmes. The problems associated with recall are more profound than is immediately evident. In spite of increasing public awareness and sophistication, recall may cause real and often prolonged anxiety to parents who find it difficult to accept that the problem was simply technical and to rid their minds of the suspicion that their infant's foibles may be related to thyroid dysfunction. Twenty per cent of parents showed persistent emotional disturbance six to 12 months after their child was recalled for retesting. ${ }^{23}$ The parents of confirmed cases may also have difficulty in accepting fully that the health of their child, never apparently ill, is dependent on the tiny daily dose of thyroxine.

Another problem attending all screening programmes is the repetitive nature of the tasks involved in collecting, processing, and testing large numbers of specimens. Increasing automation is helpful but boredom is a real threat especially in the fallow periods seen in all laboratories when the positive diagnosis rate temporarily falls. The same problem applies to the medical staff who miss the diagnostic drama and treatment thrills which so ignited William Osler.

\section{Outstanding questions}

The cause of the embryonic disturbance that results in thyroid dysgenesis remains unknown. It is remarkable that such a specific and usually isolated defect should occur with such uniform but seemingly sporadic incidence in all those areas of the world where neonatal screening has been established. Although there are hints of racial differences in incidence, Spanish and Negro infants may be under represented ${ }^{24}$ and Asian infants over represented, none have yet emerged clearly. Genetic factors are important, girls are affected 2.5 times more commonly than boys, and most identical twin pairs are concordant. Familial incidence otherwise is very rare, although there may be some general increase in thyroid disease among affected families.

The incidence of congenital and early acquired hypothyroidism among infants with Down's syndrome is high but this is not due to dysgenesis or autoimmune disease and the cause is uncertain. ${ }^{25}$ Close follow up of children with Down's syndrome is needed, especially as they seem under represented in most series.

Transplacental passage of cytotoxic antithyroid antibodies seemed in theory a likely cause but current evidence suggests this is very rarely the case. $^{26}$ Immunoglobulins, however, that block thyroid stimulating hormone induced growth of the thyroid have recently been detected by a sensitive cytochemical bioassay in some infants with sporadic congenital hypothyroidism and their mothers. ${ }^{27} \mathrm{~A}$ clue to an environmental trigger has come from the Japanese experience where an increased incidence in the summer months was noted. ${ }^{28}$

Uncertainty persists concerning the ultimate prognosis and its relation to the age of onset and severity of the fetal hypothyroidism and the postnatal delay 
in starting treatment. Does the saving of a few days, or even hours, make a difference? Does it perhaps make a difference only in those infants with the most severe and long standing fetal hypothyroidism? The evidence to date is reassuring but there may be a small minority of infants for whom treatment will be fully successful only if started before birth. If this proves to be so it will fuel the search for a means of prenatal diagnosis and treatment as a further stage in taming this disease before the ultimate goal of prevention is reached.

Another major area of uncertainty concerns the importance of the transient disorders of thyroid function, especially in preterm infants. Do these have importance in terms of lung maturation or the development of other systems? ${ }^{9}$ Once again the current evidence is reassuring.

Treatment regimens at present remain largely empirical, can these be improved?

\section{Medical Research Council congenital hypothyroid- ism register}

The register has collected data on a large cohort of cases of congenital and acquired hypothyroidism up to the age of 12 years. The progress of affected infants and carefully selected controls will be followed closely. These data, like those of the similar phenylketonuria register, will provide a unique national survey which should in time help to clarify some remaining uncertainties.

There is still far to go before congenital hypothyroidism is finally conquered or eradicated but in a decade of international activity it has been mastered in the developed world. As a result many children lead incomparably fuller lives.

\section{References}

1 Osler W. Sporadic cretinism in America. Transactions of the Congress of American Physicians and Surgeons. 1897;4:169-206.

2 Smith DW, Blizzard RM, Wilkins L. The mental prognosis in hypothyroidism of infancy and childhood. A review of 128 cases. Pediatrics 1957;19:1011-22.

${ }^{3}$ Klein AH, Meltzer S, Kenny FM. Improved prognosis in congenital hypothyroidism treated before age three months. J Pediatr 1972;81:912-3.

4 Alm J, Larsson A, Zetterstrom IR. Congenital hypothyroidism in Sweden. Incidence and age at diagnosis. Acta Paediatr Scand 1978;67:1-3

${ }^{5}$ Fisher DA, Dussault JH, Foley TP, Jr, et al. Screening for congenital hypothyroidism: results of screening one million North American infants. J Pediatr 1979;94:700-5.

6 Newborn Committee of the European Thyroid Association. Neonatal screening for congenital hypothyroidism in Europe. Acta Endocrinol 1979;90:(Suppl)223:5-29.

${ }^{7}$ Fisher DA, Klein AH. Thyroid development and disorders of thyroid function in the newborn. $N$ Engl J Med 1981;304: 702-12.

${ }^{8}$ Morreale de Escobar G, Escobar del Rey F. Brain damage and thyroid hormone. In: Burrow GN, Dussault JH, eds. Neonatal thyroid screening. New York: Raven Press, 1980:25-50.

9 Naruse H, Irie M, (eds). Neonatal screening. Proceedings of the second international conference on neonatal thyroid screening. Amsterdam: Excerpta Medica 1983:1-188.

${ }^{10}$ Price DA, Ehrlich RM, Walfish PG. Congenital hypothyroidism. Clinical and laboratory characteristics in infants detected by neonatal screening. Arch Dis Child 1981;56:845-51.

11 Dussault JH, Morissette J. Higher sensitivity of primary thyrotropin in screening for congenital hypothyroidism: a myth? $J$ Clin Endocrinol Metab 1983;56:849-55.

12 Fisher DA. Medical management of suspected cases of congenital hypothyroidism. In: Burrow GN, Dussault JH, eds. Neonatal thyroid screening. New York: Raven Press, 1980:237-45.

${ }^{13}$ New England Congenital Hypothyroidism Collaborative. Characteristics of infantile hypothyroidism discovered on neonatal screening. J Pediatr 1984;104:539-44.

14 Desai M, Irani AJ, Patil K, Pandya CS. The importance of reverse triidothyronine in hypothyroid children on replacement treatment. Arch Dis Child 1984;59:30-5.

15 Sato T, Suzuki Y, Taketani T, Ishiguro K, Nakajima H. Agerelated change in pituitary threshold for TSH release during thyroxine replacement therapy for cretinism. J Clin Endocrinol Metab 1977;44:553-9.

${ }^{16}$ Hulse JA, Grant DB, Jackson D, Clayton BE. Growth, development, and reassessment of hypothyroid infants diagnosed by screening. Br Med $J$ 1982;284:1435-9.

17 Glorieux J, Dussault JH, Letarte J, Gyda H, Morissette J. Preliminary results on the mental development of hypothyroid infants detected by the Quebec screening program. $J$ Pediatr 1983;102:19-22.

${ }^{18}$ Money J, Clark FC, Beck J. Congenital hypothyroidism and IQ increase: a quarter century follow-up. J Pediatr 1978;93:432-4.

19 La Berge C. Organisation and cost benefits of mass screening programs. In: Burrow GN, Dussault JH, eds. Neonatal thyroid screening. New York: Raven Press, 1980:189-97.

20 New England Regional Screening Program. Pitfalls in screening for neonatal hypothyroidism. Pediatrics 1982;70:16-20.

21 Gendrel D, Feinstein M-C, Grenier J, et al. Falsely elevated serum thyrotropin (TSH) in newborn infants: transfer from mother to infants of a factor interfering in the TSH immunoassay. J Clin Endocrinol Metab 1981;52:62-5.

${ }^{22}$ Lazarus $\mathrm{JH}$, John $\mathrm{R}$, Ginsberg $\mathrm{J}$, et al. Transient neonatal hyperthyrotropinaemia: a serum abnormality due to transplacentally acquired antibody to thyroid stimulating hormone. Br Med J 1983;286:592-4.

${ }^{23}$ Bodegard G, Fyrok K, Larsson A. Psychological reactions in 102 families with a newborn who has a falsely positive screening test for congenital hypothyroidism. Acta Paediatr Scand [Suppl] 1983;304:1-21.

${ }^{24}$ Brown AL, Fernhoff PM, Milner J, McEwen C, Elsas LS Racial differences in the incidence of congenital hypothyroidism. J Pediatr 1981;99:934-6.

25 Fort $\mathrm{P}$, Lifschitz F, Bellisario R, et al. Abnormalities of thyroid function in infants with Down syndrome. $J$ Pediatr 1984;104:545-9.

26 Dussault JH, Letarte J, Guyda H, Laberge C. Lack of influence of thyroid antibodies on thyroid function in the newborn infant and on a mass screening program for congenital hypothyroidism. J Pediatr 1980;96:385-9.

${ }^{27}$ Van der Gaag RD, Drexhage HA, Dussault JH. Role of maternal immunoglobulins blocking TSH-induced thyroid growth in sporadic forms of congenital hypothyroidism. Lancet $1985 ; \mathbf{i}: 246-50$.

28 Miyai K, Ichihara K, Amino N, et al. Seasonality of birth in sporadic cretinism. Early Hum Dev 1978;3:85-8.

Correspondence to Dr N D Barnes, Department of Paediatrics, Addenbrooke's Hospital, Cambridge CB2 2QQ. 